\title{
A Delayed Pseudoaneurysm Rupture in Low-Grade Splenic Injury
}

\author{
Chan Yong Park', So Ra Ahn', Wu Seong Kang1, Sang Hyun Seo², Sung Nam Moon \\ ${ }^{1}$ Department of Trauma Surgery, ${ }^{2}$ Department of Radiology, Wonkwang University, Jeonbuk, Korea
}

Delayed rupture of the spleen is one of the complications of splenic injury not managed surgically. However, delayed pseudoaneurysm rupture in low-grade splenic injury is extremely rare. I report the case of a 74-year-old man with low-grade splenic injury who suffered delayed pseudoaneurysm rupture. The patient was admitted with low-grade splenic injury (grade II Organ Injury Scale [OIS] score according to the American Association for the Surgery of Trauma [AAST]). On chest computed tomography on day 6, a pseudoaneurysm approximately $2.5 \times 1.5 \times 2.5 \mathrm{~cm}$ in size appeared in the spleen; however, the physician missed this finding at that time. On day 13, chest computed tomography confirmed pseudoaneurysm rupture. The ruptured pseudoaneurysm was successfully treated with transcatheter arterial embolization. This rare case is reported to describe the entire process from occurrence to rupture of the splenic pseudoaneurysm in a low-grade splenic injury.

(Trauma Image Proced 2019(1):15-18) Key Words: Delayed rupture, Splenic injury, Pseudoaneurysm, Organ Injury Scale grade II, Transcatheter arterial embolization

\section{CASE}

A 74-year-old man was referred to our emergency department from another hospital after meeting with a traffic accident. At initial measurement, his blood pressure was 100/70 $\mathrm{mm} \mathrm{Hg}$; his pulse rate, 91 beats/min; his respiration rate, 22 breaths/min; his body temperature, $37.0^{\circ} \mathrm{C}$; and his oxygen saturation, $90 \%$. Initial abdomen computed tomography (CT) showed American Association for the Surgery of Trauma (AAST) grade II splenic injury according to the Organ Injury Scale (OIS) score, and a tiny pseudoaneurysm was suspected (Fig. 1.). On chest CT on day 6 after admission, a pseudoaneurysm approximately $2.5 \times 1.5 \times$ $2.5 \mathrm{~cm}$ in size appeared in the spleen (Fig. 2.); however, the physician missed the finding at that time. The hemoglobin level was maintained well at $11.0 \mathrm{~g} / \mathrm{dL}$ on day 8 but decreased to $9.1 \mathrm{~g} / \mathrm{dL}$ on day 9 and increased to 10.6 after transfusion of $2 \mathrm{U}$ of packed red blood cells. On the day 13, chest CT confirmed pseudoaneurysm rupture and perisplenic hematoma. At the site of the pseudoaneurysm, CT showed no filling with contrast medium, and an area of low density was seen (Fig. 3.). Splenic arteriography was performed, and a contrast defect was found at the position corresponding to the area of low density on CT. Although no contrast medium extravasation was visible, the superior segmental branch was embolized with absorbable gelatin powder (Gelfoam) to prevent possible delayed bleeding (Fig. 4.). On abdomen CT performed on day 19, the perisplenic

Received: February 15, 2019 Revised: May 12, 2019 Accepted: May 12, 2019

Correspondence to: Chan Yong Park, MD, Department of Trauma Surgery, Wonkwang University Hospital, Jeonbuk, Korea Tel: 82-63-859-2602, Fax: 82-63-859-2029, E-mail: wkafyddl@hanmail.net

Copyright (c) 2019 Korean Association for Research, Procedures and Education on Trauma. All rights reserved.

@This is an open-access article distributed under the terms of the Creative Commons Attribution Non-Commercial License (http://creativecommons.org/ licenses/by-nc/4.0) which permits unrestricted noncommercial use, distribution, and reproduction in any medium, provided the original work is properly cited 


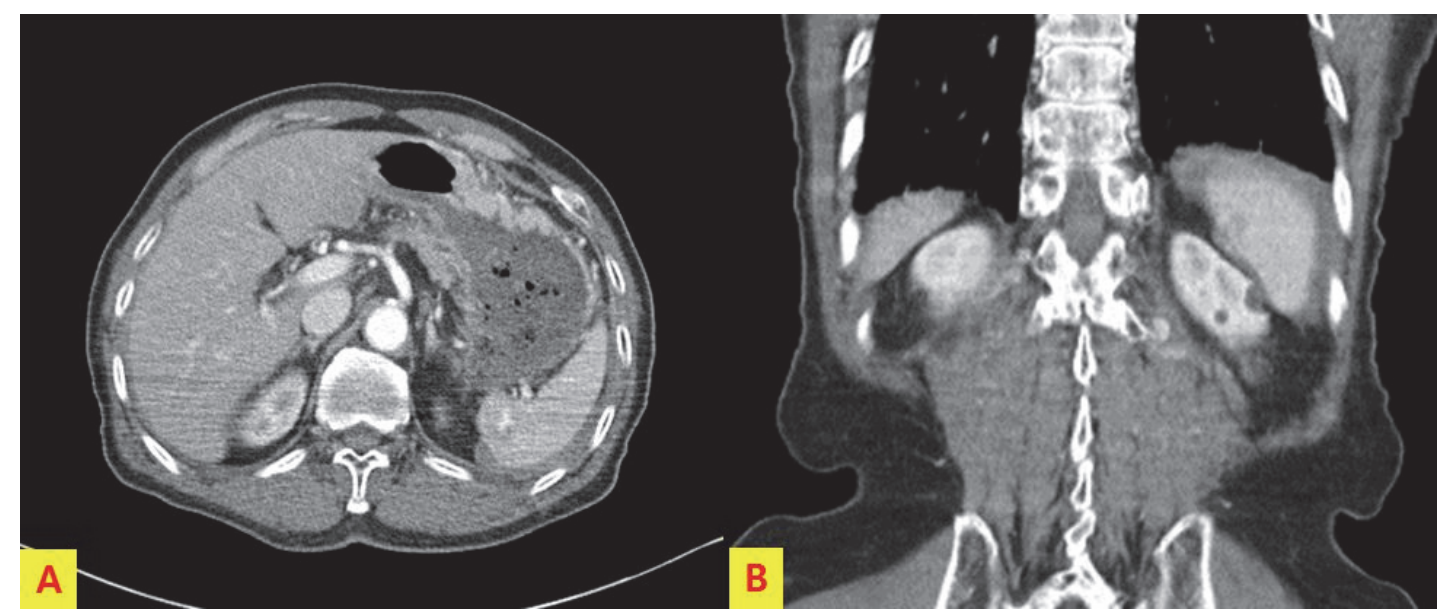

Fig. 1. Initial abdomen CT showed AAST OIS grade II splenic injury, and tiny pseudoaneurysm was suspected.

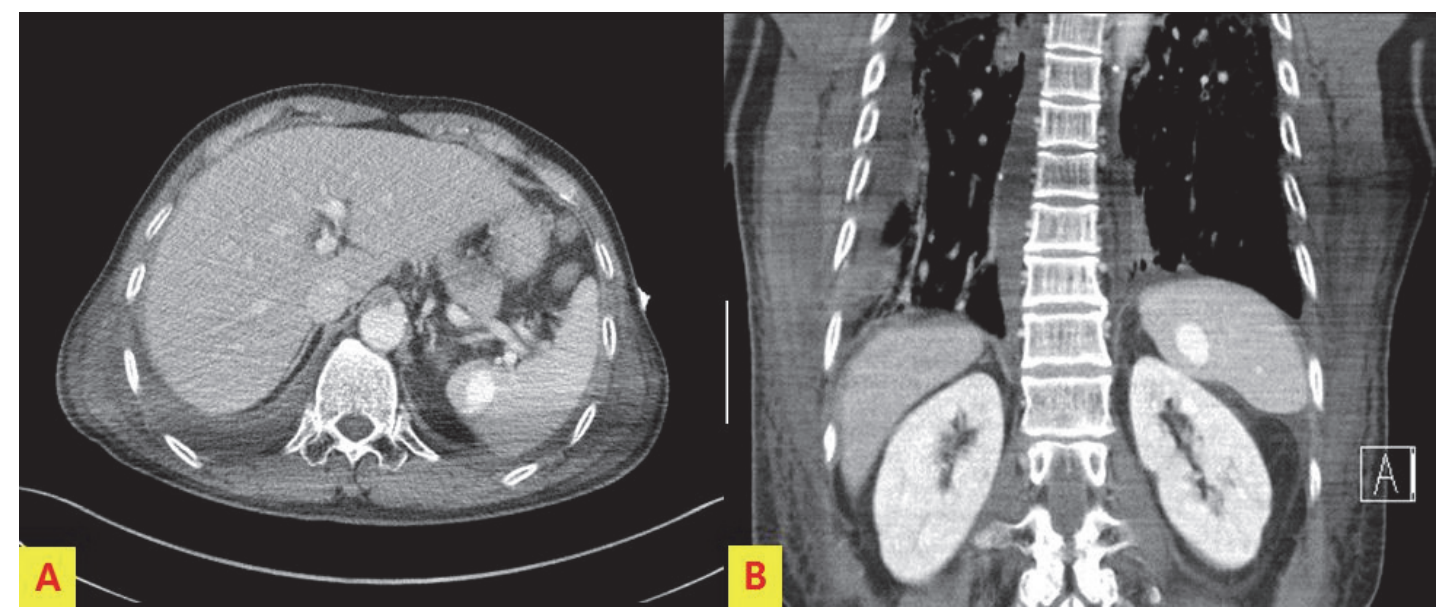

Fig. 2. On chest CT at $6^{\text {th }}$ day, approximately $2.5 \times 1.5 \times 2.5 \mathrm{~cm}$ sized pseudoaneurysm was appeared.

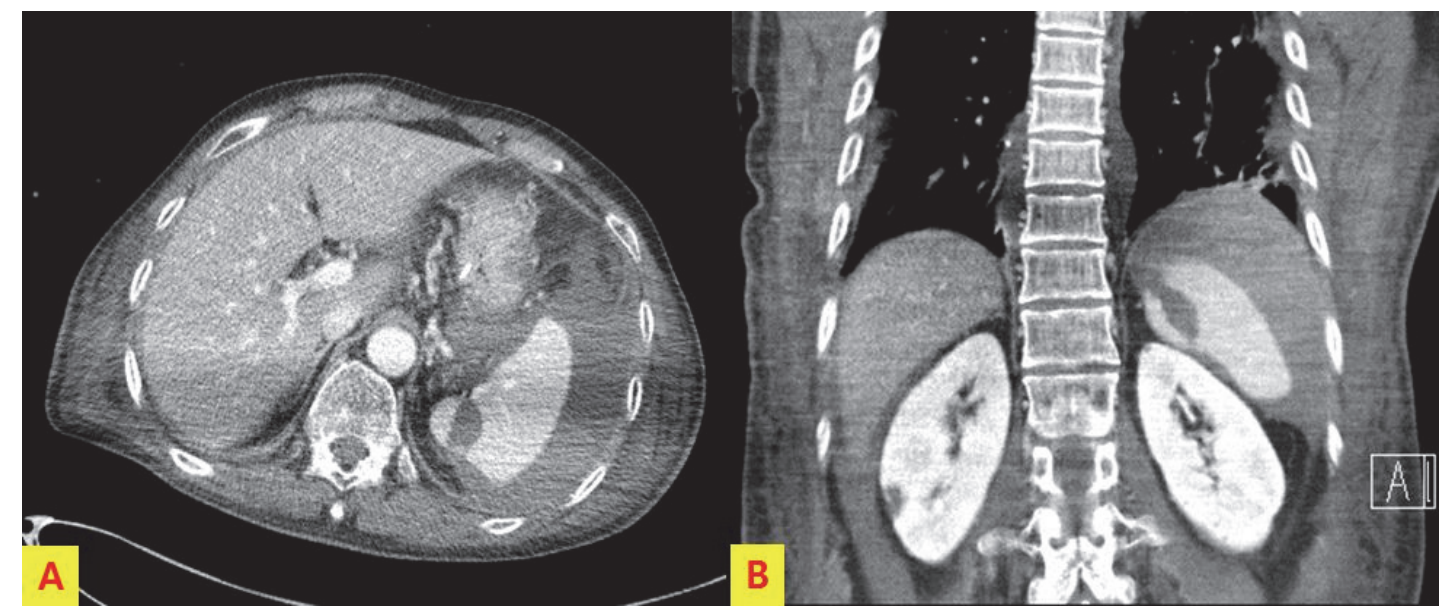

Fig. 3. On the $13^{\text {th }}$ day, chest CT confirmed pseudoaneurysm rupture and perisplenic hematoma. At the site where pseudoaneurysm was present, there was no contrast filling and low density finding was seen. 


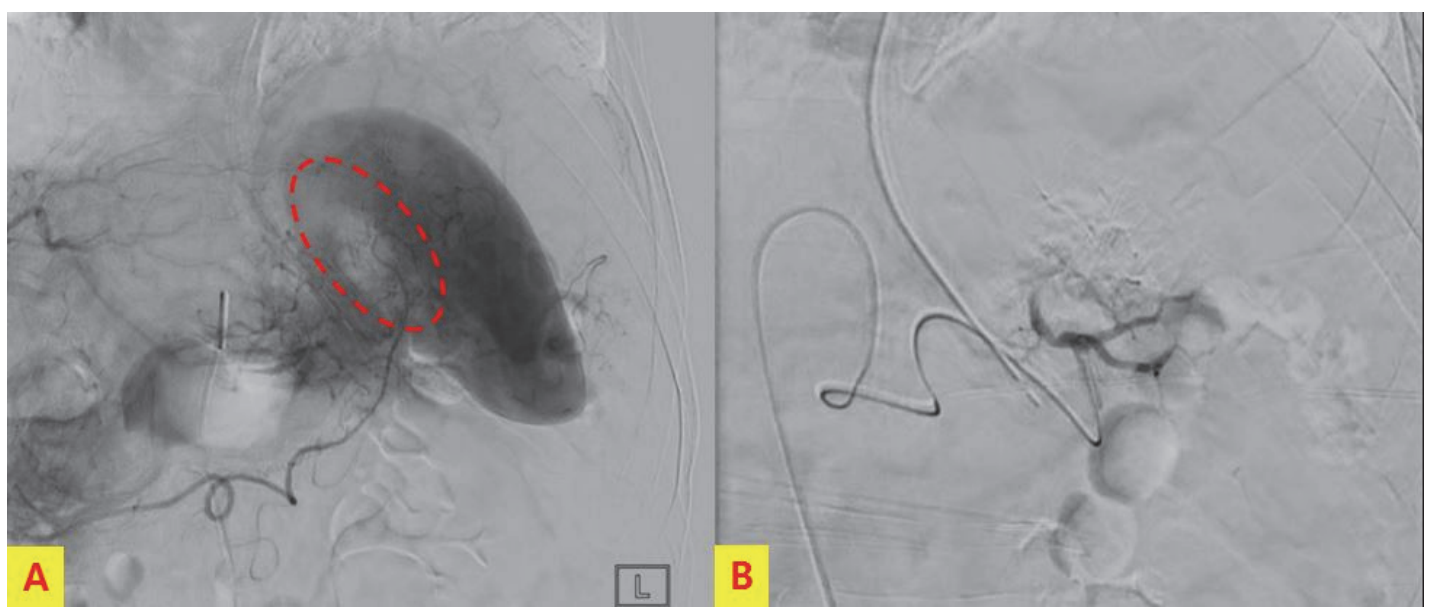

Fig. 4. Splenic arteriography was performed and a contrast defect (empty oval with red dotted line) was found at the position correlated with the area of the low density finding after pseudoaneurysm formation on CT (A). The superior segmenta branch was embolized with gelfoam (B).

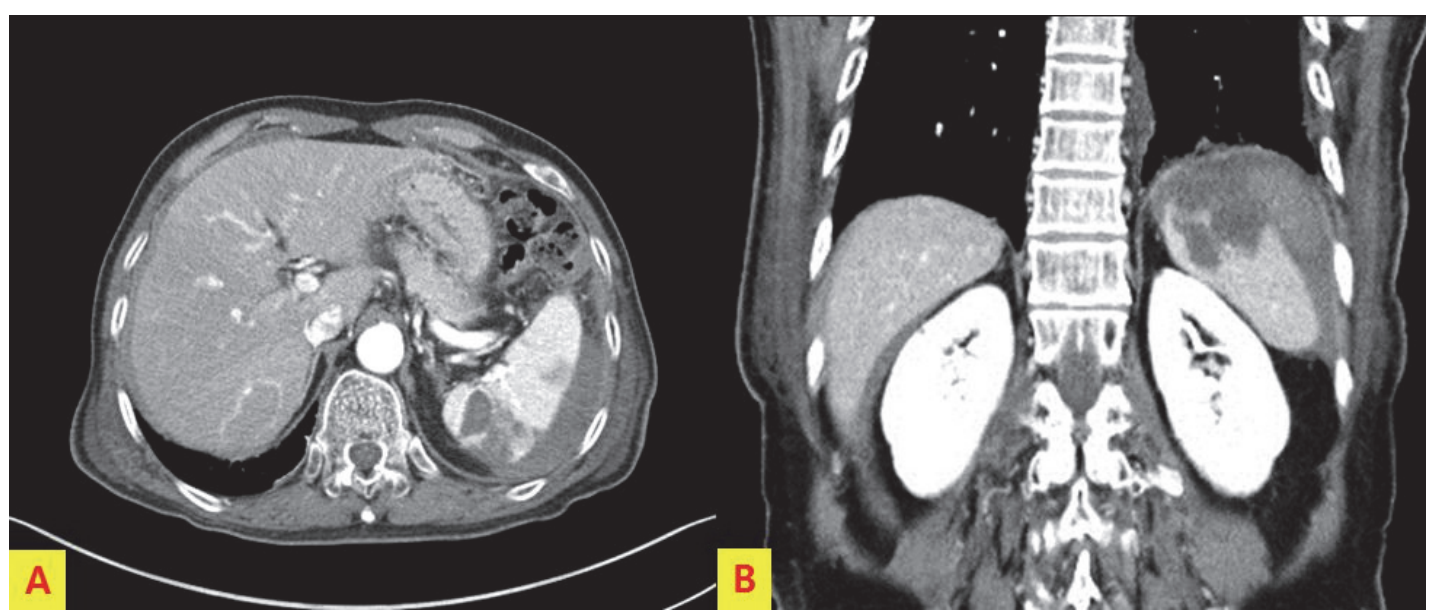

Fig. 5. On abdomen CT performed on $19^{\text {th }}$ day of admission, perisplenic hematoma decreased and no bleeding or pseudoaneurysm was observed.

hematoma was smaller, and no bleeding or further pseudoaneurysm was observed (Fig. 5.).

\section{DISCUSSION}

Delayed splenic rupture following nonoperative management (NOM) in splenic injury is a rare complication. Resteghini et al. (1) reported a case of splenectomy due to delayed splenic rupture after 2 months in a blunt abdominal trauma patient who had only minimal splenic contusion at the initial examination. Muroya et al. (2) studied the delayed formation of splenic pseudoaneurysm
(SPA) in patients with NOM in blunt splenic injury. In this study, delayed formation of SPA on follow up enhanced CT was confirmed at $15.4 \%$, and timing was mean (SD) of 4.6 (2.1) hospital days (range, 1-8 days) after admission. About half of the SPA was occluded spontaneously without therapeutic intervention. They suggested that follow up enhanced CT for approximately 1 week after splenic injury may be useful for detecting delayed SPA formation. Splenic Pseudoaneurysm can be diagnosed when turbulent arterial flow is observed with color Doppler sonography in anechoic lesion on sonography (3). In the guideline of the World Society of 
Emergency Surgery for splenic trauma, the observation failure risk (OFR) computed tomographic (CT) scan criteria was included. Here, CT scan signs associated with high OFR are blush, pseudoaneurysm, OIS grade III with a large hemoperitoneum, and OIS grade IV or V (4). Brault-Noble et al. (5) classified and treated 208 blunt splenic injuries using OFR CT scan criteria. NOM was performed in $161(77 \%)$ and $49(30 \%)$ had high OFR CT scan criteria. Thirteen patients (8\%) experienced observation failure. In this study, high OFR CT scan criteria (odds ratio, 11.95: 95\% confidence interval, 2.5-47.5) and the age of 50 years or older (odds ratio, 33.9: 95\% confidence interval, 6.2-185.5) were independent factors related to observation failure. To reduce the morbidity and mortality associated with splenic trauma and subsequent delayed rupture, it is important for practitioner to have working knowledge of risk factors, diagnostic studies and interventions (6). In the present case, splenic injury was OIS II, and very small pseudoaneurysm was present on initial $\mathrm{CT}$ and increased to $2.5 \mathrm{~cm}$ on $\mathrm{CT}$ at 6th day of admission. On day 9, hemoglobin decreased by 1.9 , and it was suspected to have been ruptured. Actually, splenic pseudoaneurysm rupture was confirmed by $\mathrm{CT}$ on day 13. Therefore, even the small splenic pseudoaneurysm need a careful and planned follow up plan.

\section{Conflict of Interest Statement}

None of authors has a conflict of interest

\section{REFERENCE}

1. Resteghini N, Nielsen J, Hoimes ML, Karam AR. Delayed splenic rupture presenting 70 days following blunt abdominal trauma. Clin Imaging 2014;38(1):73-4.

2. Muroya T, Ogura H, Shimizu K, Tasaki O, Kuwagata Y, Fuse $\mathrm{T}$, et al. Delayed formation of splenic pseudoaneurysm following nonoperative management in blunt splenic injury: multi-institutional study in Osaka, Japan. J Trauma Acute Care Surg 2013;75(3):417-20.

3. Fitoz S, Atasoy C, Dusunceli E, Yagmurlu A, Erden A, Akyar S. Post-traumatic intrasplenic pseudoaneurysms with delayed rupture: color Doppler sonographic and CT findings. J Clin Ultrasound 2001;29(2):102-4.

4. Coccolini F, Montori G, Catena F, Kluger Y, Biffl VV, Moore EE, et al. Spenic trauma: WSES classification and guidelines for adult and pediatric patients. World J Emerg Surg 2017;18:12:40.

5. Brault-Noble G, Charbit J, Chardon P, Barral L, Guillon F, Taourel $\mathrm{P}$, et al. Age should be considered in the decision making of prophylactic splenic angioembolization in nonoperative management of blunt splenic trauma: a study of 208 consecutive civilian trauma patients. J Trauma Acute Care Surg 2012;73(5):1213-20.

6. Ruffolo DC. Delayed splenic rupture: understanding the threat. J Trauma Nurs 2002;9(2):34-40. 